\title{
Cytokine storms in infectious diseases
}

\author{
John R. Teijaro ${ }^{1}$
}

Received: 4 May 2017 / Accepted: 8 May 2017

(C) Springer-Verlag Berlin Heidelberg 2017

The term "cytokine storm" has been adopted in the past several decades as a phrase to describe the aberrant production of soluble mediators and the accompanying immunopathology that ensues following severe viral and bacterial infections. However, "cytokine storm" was initially coined in the early 1990 s to characterize the pathological condition that accompanied organ transplantation, an allogenic response to foreign tissue called graft vs host disease $[1,2]$. More recently, aberrant immune responses and cytokine production have been associated with the pathogenesis of multiple disease states ranging from viral infection to neurological disorders [3]. Despite a definitive link of cytokine and chemokine levels with morbidity and mortality following infectious insults, no effective therapeutic treatments or modalities have been developed to quell the pathology associated with cytokine storm. In fact, the apparent intractability of tested therapies to subdue pathology has led some to postulate whether robust cytokine and chemokine production observed during these disease states is directly causal to the clinical manifestations. One major reason for this is that the kinetics, cellular sources, and cytokine milieu that mediate disease pathology remain poorly understood. This special issue of Seminars in Immunopathology entitled "Cytokine Storms in Infectious Diseases" focuses on the role of cytokines and other soluble mediators and their function in promoting pathology during

This article is a contribution to the special issue on Cytokine Storm in Infectious Diseases - Guest Editor: John Teijaro

John R. Teijaro

teijaro@scripps.edu

1 Department of Immunology and Microbial Science, The Scripps Research Institute, La Jolla, San Diego, CA 92037, USA infectious diseases and highlights emerging data that may point the way toward novel therapeutic interventions.

The immune system secretes soluble mediators to instruct both innate and adaptive immune responses to respond appropriately to infectious insults. Thus, cytokines and chemokines play a vital role in maintaining a healthy state through efficient control of invading microbes. Clark and Vissel review the history of cytokine storm and highlight the roles cytokines play in instructing proper immune responses as well as mediating determinantal immunopathology [4]. The primary focus is on the role of two historical soluble mediators, Tumor necrosis factor- $\alpha$ (TNF- $\alpha$ ) and Interleuken-1 (IL-1), in multiple acute and chronic disease states. Moreover, the authors review the role non-resolving persistent inflammation plays in neurodegenerative disease states. Finally, the authors emphasize that understanding how the varying degrees of acuteness and chronicity of cytokine storms influence various diseases will be crucial in developing novel therapies to control associated pathologies.

The link between inflammatory cytokine/chemokine production and clinical disease following sepsis in patients has been recognized for several decades [5, 6]. Despite this strong correlation, no effective therapies targeting the inflammatory response have been generated to alleviate sepsis associated morbidity and mortality. Chousterman et al. review the contribution of various cytokines to the pathophysiology of sepsis [7]. Importantly, the authors emphasize that the kinetics of cytokine production following sepsis is likely to dictate successful therapeutic intervention. Moreover, they stress that patient sub-setting will be crucial for administering effective therapies to diverse patient populations. Finally, the authors underscore the importance of increased vascular permeability following sepsis and highlight treatments that target endothelial cell function as potential therapeutic options for a subset of sepsis patients. 


\section{Respiratory viral infections}

Elevated cytokine and chemokine production has been continuously associated with poor clinical outcome and pathogenesis during respiratory viral infections in humans and animal models [8-10]. Perlman and Thomas et al. review in detail the role various cytokines, chemokines and innate immune cells play in promoting pathology or protection during two clinically important respiratory viruses: coronaviruses $(\mathrm{CoV})$ and influenza viruses, respectively $[11,12]$. Specifically, Channappanavar and Perlman highlight how pathogenic $\mathrm{CoV}$ antagonize early type 1 interferon signaling which promotes rapid virus replication, setting the stage for an exacerbated secondary inflammatory environment marked by elevated cytokines/chemokines and accumulation of inflammatory monocytes/macrophages and neutrophils in the infected lung [11]. Moreover, Guo and Thomas emphasize the emerging roles of type 2 cytokines such as amphiregulin in dampening inflammation and promoting tissue repair [12]. Both reviews discuss the current experimental anti-inflammatory strategies being tested in vivo in animal models including Sphingosine 1 phosphate modulators, COX inhibitors, PPAR (peroxisome proliferator-activated receptor) agonists and modulation of production/signaling of oxidized phospholipids, and their potential as future therapeutic options to ameliorate cytokine storm pathology during respiratory viral infection.

\section{Pathogenesis of viral hemorrhagic fevers}

The recent deadly outbreak of Ebola virus in West Africa which killed over 11,000 people sparked fear into millions of people in the USA when the virus made its way to America. While Ebola virus pathology is the result of multiple factors, excessive inflammatory responses are a significant feature following infection. Probably the most notable symptom is hemorrhage which can occur at varying degrees of severity following Ebola virus infection. The aberrant inflammatory response that accompanies Ebola virus infection, termed viral hemorrhagic fever (VHF), can be caused by multiple RNA viruses, including arenaviruses, bunyaviruses, filoviruses, and flaviviruses. Basler reviews the linkages between excessive inflammatory responses, hemorrhage and disseminated intravascular coagulation in the pathological manifestation of VHF in humans and non-human primates [13]. The review highlights potential sources of inflammatory cytokines during multiple VHF infections and points out that while dendritic cells are targets of viral replication by multiple VHF viruses, these cells secrete minimal amounts of cytokines/chemokines while monocytes and macrophages appear to be significant sources of inflammatory cytokines during these infections. The author highlights that the most relevant cell types and signaling pathways responsible for disease pathology in vivo have yet to be determined and understanding how the cytokine storm progresses in vivo will likely yield optimal therapeutics to subdue pathology associated with these infections.

\section{Role of cytokine storm in dengue virus pathogenesis}

Dengue virus infection and disease is endemic to the tropical and sub-tropical regions of the globe, largely due to the vector borne nature of its infection cycle where primary mosquito infection is necessary for transmission to humans. However, the recent warming of the globe has begun to push mosquitos that transmit dengue further north, making parts of the USA and southern European nations more likely to experience dengue infection. Although severe dengue infection occurs in a minority of patients, the accompanying dengue hemorrhagic fever (DHF) is characterized by increased vascular permeability, hemorrhage, and organ failure. In this series, Rothman and colleagues review how both innate and adaptive immune responses contribute to promoting the severe manifestation of DHF [14]. The authors also review specific key cytokines and chemokines, namely TNF- $\alpha$, vascular endothelial growth factors (VEGF-A), IL-6, IL-10, IL-8, CCL2, and CXCL10, and how their production promotes clinical presentation of DHF. However, the authors point out that efficacious therapies for DHF will likely require targeting proteins that suppress multiple inflammatory pathways in humans. Thus, due to the absence of good animal models, there is an urgent need for welldesigned patient studies coupled to competent bioinformatic analysis to identify reliable factors and pathways that promote DHF pathology.

\section{Strategies to mitigate cytokine storm and future perspectives}

Current research is illuminating the cellular and molecular contributors of cytokine storm in multiple disease states. Based on recent developments in the field, new therapies will likely move away from targeting single soluble mediators and focus more generally on inflammatory cascades. However, what signaling cascades are targeted will depend both on the infection as well as the targeted patient population. Current experimental anti-inflammatory strategies being tested in animal models include sphingosine 1 phosphate modulators [15, 16], COX inhibitors, Slit-Robo4 pathway modulators [17], PAR2 agonists [18], modulation of production/signaling of oxidized phospholipids [19], and TLR4 antagonists [20]. However, it is clear from ongoing studies that global blunting, not ablation, of inflammatory mediators will likely be required to ameliorate pathology associated with cytokine storm as one 
must balance suppression of immune pathology with proper control of the infectious agent.

\section{References}

1. Ferrara JL (1993) Cytokine dysregulation as a mechanism of graft versus host disease. Curr Opin Immunol 5:794-799

2. Ferrara JL, Abhyankar S, Gilliland DG (1993) Cytokine storm of graft-versus-host disease: a critical effector role for interleukin-1. Transplant Proc 25:1216-1217

3. Tisoncik JR, Korth MJ, Simmons CP, Farrar J, Martin TR, Katze MG (2012) Into the eye of the cytokine storm. Microbiol Mol Biol Rev 76:16-32

4. Clark IA, Vissel B (2017) The meteorology of cytokine storms, and the clinical usefulness of this knowledge. Semin Immunopathol. doi:10.1007/s00281-017-0628-y

5. Bone RC, Balk RA, Cerra FB, Dellinger RP, Fein AM, Knaus WA, Schein RM, Sibbald WJ (1992) Definitions for sepsis and organ failure and guidelines for the use of innovative therapies in sepsis. The ACCP/SCCM Consensus Conference Committee. American College of Chest Physicians/Society of Critical Care Medicine. Chest 101:1644-1655

6. Bone RC, Sibbald WJ, Sprung CL (1992) The ACCP-SCCM consensus conference on sepsis and organ failure. Chest 101:14811483

7. Chousterman BG, Swirski FK, Weber GF (2017) Cytokine storm and sepsis disease pathogenesis. Semin Immunopathol. doi:10. 1007/s00281-017-0639-8

8. Cilloniz C, Shinya K, Peng X, Korth MJ, Proll SC, Aicher LD, Carter VS, Chang JH, Kobasa D, Feldmann F, Strong JE, Feldmann H, Kawaoka Y, Katze MG (2009) Lethal influenza virus infection in macaques is associated with early dysregulation of inflammatory related genes. PLoS Pathog 5:e1000604

9. de Jong MD, Simmons CP, Thanh TT, Hien VM, Smith GJ, Chau TN, Hoang DM, Chau NV, Khanh TH, Dong VC, Qui PT, Cam BV, Ha do Q, Guan Y, Peiris JS, Chinh NT, Hien TT, Farrar J (2006) Fatal outcome of human influenza A (H5N1) is associated with high viral load and hypercytokinemia. Nat Med 12:1203-1207
10. Zhang Y, Li J, Zhan Y, Wu L, Yu X, Zhang W, Ye L, Xu S, Sun R, Wang Y, Lou J (2004) Analysis of serum cytokines in patients with severe acute respiratory syndrome. Infect Immun 72:4410-4415

11. Channappanavar R, Perlman S (2017) Pathogenic human coronavirus infections: causes and consequences of cytokine storm and immunopathology. Semin Immunopathol. doi:10.1007/s00281017-0629-x

12. Guo XJ, Thomas PG (2017) New fronts emerge in the influenza cytokine storm. Semin Immunopathol. doi:10.1007/s00281-0170636-y

13. Basler CF (2017) Molecular pathogenesis of viral hemorrhagic fever. Semin Immunopathol. doi:10.1007/s00281-017-0637-x

14. Srikiatkhachorn A, Mathew A, Rothman AL (2017) Immunemediated cytokine storm and its role in severe dengue. Semin Immunopathol. doi:10.1007/s00281-017-0625-1

15. Teijaro JR, Walsh KB, Cahalan S, Fremgen DM, Roberts E, Scott F, Martinborough E, Peach R, Oldstone MB, Rosen H (2011) Endothelial cells are central orchestrators of cytokine amplification during influenza virus infection. Cell 146:980-991

16. Oldstone MB, Teijaro JR, Walsh KB, Rosen H (2013) Dissecting influenza virus pathogenesis uncovers a novel chemical approach to combat the infection. Virology 435:92-101

17. London NR, Zhu W, Bozza FA, Smith MC, Greif DM, Sorensen LK, Chen L, Kaminoh Y, Chan AC, Passi SF, Day CW, Barnard DL, Zimmerman GA, Krasnow MA, Li DY (2010) Targeting Robo4-dependent Slit signaling to survive the cytokine storm in sepsis and influenza. Sci Transl Med 2:23ra19

18. Nhu QM, Shirey K, Teijaro JR, Farber DL, Netzel-Arnett S, Antalis TM, Fasano A, Vogel SN (2010) Novel signaling interactions between proteinase-activated receptor 2 and Toll-like receptors in vitro and in vivo. Mucosal Immunol 3:29-39

19. Tam VC, Quehenberger O, Oshansky CM, Suen R, Armando AM, Treuting PM, Thomas PG, Dennis EA, Aderem A (2013) Lipidomic profiling of influenza infection identifies mediators that induce and resolve inflammation. Cell 154:213-227

20. Shirey KA, Lai W, Scott AJ, Lipsky M, Mistry P, Pletneva LM, Karp CL, McAlees J, Gioannini TL, Weiss J, Chen WH, Ernst RK, Rossignol DP, Gusovsky F, Blanco JC, Vogel SN (2013) The TLR4 antagonist Eritoran protects mice from lethal influenza infection. Nature 497:498-502 